\title{
ATteLL|E
}

\section{Comportamento e distribuição das chuvas na Região de São Carlos/SP a partir de dados de Estações Climatológicas no período de 1993-2014.}

\author{
Comportamento y distribución de las lluvias en la Región de \\ São Carlos/SP por datos de Estaciones Climatológicas en el \\ período de 1993-2014.

\section{Behavior and dsitribution of rainfall in the Region of São Carlos/SP from Climatological Stations data, 1993-2014.}

\author{
Rafael Grecco Sanches \\ Universidade de São Paulo \\ rafagsanches@usp.br \\ Francisco Arthur da Silva Vecchia \\ Universidade de São Paulo \\ fvecchia@usp.br \\ Paulo Henrique de Souza \\ Universidade Federal de Alfenas \\ phsouza31@hotmail.com
}

\begin{abstract}
Resumo
As alterações climáticas, em diferentes escalas espaciais e temporais, alicerçam diversas pesquisas em climatologia a fim de compreender a dinâmica climatológica, bem como os atributos climáticos em tal processo. As chuvas, nesse sentido, representam a complexidade no entendimento da dinâmica atmosférica, uma vez que essas apresentam sua gênese apoiadas em diferentes processos atmosféricos. O presente estudo compreende o comportamento das chuvas na região de São Carlos a partir de dados diários de chuvas nas estações climatológicas na região de São Carlos/SP na série histórica de 1993 a 2014. Foram determinados os cálculos dos índices propostos por Zhang e Yang (2004) de RX 1 dia (máximo acumulado em um único dia), SDII (índice simples de intensidade), DCU (dias consecutivos úmidos), DCS (dias consecutivos secos) e PRCPTOT (precipitação total nos dias úmidos), pelo software R com script RClimdex. Os dados calculados apresentaram grande flutuação ao longo da série histórica 1993-2014 para todos os índices adotados, o que configura a presença de anos abaixo e acima da média para cada
\end{abstract}


índice, bem como aponta para o dinamismo climático regional com os valores diários das chuvas junto ao planejamento regional.

Palavras-chave: Índices, Climatologia Estatística, Chuvas, RClimdex.

\begin{abstract}
Resumen
Los cambios climáticos en diferentes escalas espaciales y temporalis contribuyen en diversas investigaciones en climatología para comprender la dinámica climatológica, así como los atributos climáticos en proceso. Las lluvias en ese sentido representan la complejidad en el entendimiento de la dinámica atmosférica una vez que éstas presentan su génesis apoyadas en diferentes procesos atmosféricos. El presente estudio comprende el comportamiento de las lluvias en la región de São Carlos a partir de datos diarios de lluvias en las estaciones climatológicas en la región de São Carlos / SP en la serie histórica de 1993 a 2014. Se determinaron los cálculos de los índices propuestos por Zhang y Yang (2004): RX 1 día (máximo acumulado en un solo día), SDII (índice simple de intensidad), DCU (días consecutivos húmedos), DCS (días consecutivos secos) y PRCPTOT (precipitación total en los días húmedos) por medio de software R y script RClimdex. Los datos calculados presentaron gran fluctuación en la serie histórica 1993-2014 y todos los índices adoptados. Esto se configura la presencia de años abajo y por encima de la media para cada índice así como al dinamismo climático regional en los valores diarios de las lluvias a la planificación regional.
\end{abstract}

Palabras Clave: Índices, Climatología Estadística. Lluvias, RClimdex.

\begin{abstract}
The climate change, in different spatial-temporal scales, consolidates several climatology researches in order to comprehend the climatologic dynamics, as well as climate attributes in such process. Rain represents complexity on the atmospheric dynamic understanding, once it presents its genesis based on different atmospheric processes. With that, the following study comprehends the rain behavior in the region of São Carlos/SP according to daily rain data in the climatological stations in this region from the historical series from 1993 to 2014. In this study, the bibliographic revision was performed concerning the rain genesis for Brazil and for the state of São Paulo, apart from the determination of indices proposed by Zhang and Yang (2004), RX 1 day (maximum accumulated in a single day), SDII (single strength index), DCU (consecutive wet days), DCS (consecutive dry days), PRCPTOT (total precipitation in wet days) and index calculus through the software $\mathrm{R}$ with the script Rclimdex 1.1. The calculated data present a great floating along the historical series 1993-2014 for all the admitted index, which sets the presence of years below and above the average for each index and point to regional climate dynamics with daily values of rainfall by the regional planning.
\end{abstract}

Keywords: Indices, Statistical Climatology, Rain, RClimdex.

\title{
Introdução
}

Os estudos climáticos assumem um papel de grande importância junto às relações entre homem e ambiente. Além de complexas, tais relações se manifestam de forma distinta espacial e temporalmente. Para tanto, as variabilidades temporais e associações com outros elementos naturais contribuem para uma melhor compreensão da estrutura do espaçoambiente, bem como de sua organização funcional (MONTEIRO, 1976; COELHO et al, 2016). 
Um dos mecanismos utilizados para a interpretação de dados climatológicos é a análise estatística que, por sua vez, permite avaliar um sistema climático a partir de inúmeras informações (precipitação pluviométrica, temperatura do ar, pressão atmosférica, etc.) e, dessa forma, permite a observação de pressupostos quanto a mudanças num sistema climático (ZWIERS; VON STORCH, 2004).

Diante de diferentes conceituações sobre o que é mudança climática, Santos (2014) a caracteriza como mudanças nas variáveis climáticas pela média e valores extremos, bem como na forma de distribuição estatística. O autor afirma, que as análises de séries históricas, anuais e sazonais, das variáveis climáticas, podem ser utilizadas como indicadores de avaliação das mudanças no regime dos climas, tendo em vista a temperatura e a precipitação pluviométrica como principais parâmetros.

As chuvas assumem papel de grande destaque na compreensão do clima, bem como podem ser consideradas como principal elemento de análise na organização e no planejamento do território e do ambiente diante de seu elevado grau de interferência, impacto e repercussão espaço-temporal (SANTA'ANNA NETO et al., 2000).

Na região tropical, a variabilidade das chuvas é mais sazonal do que nas áreas extratropicais, uma vez que em baixas latitudes, essas são controladas pelo eixo norte-sul dos ventos, bem como associadas à convergência e divergência de tais ventos (AYOADE, 2002).

No sudeste do Brasil, fenômenos típicos de verão da América do Sul, como a convergência de ventos e a persistência de nebulosidade sentido noroeste-sudeste persiste e implica em elevados volumes de chuvas (STEINKE, 2012).

$\mathrm{O}$ aquecimento de algumas áreas da América do Sul acarreta uma área ascendente na troposfera e de divergência de ar próximo a tropopausa denominada Alta da Bolívia (AB), que implica na elevação da umidade em níveis próximos à superfície. Com isso, bem como por sua interação com o avanço de um sistema frontal notam-se dias consecutivos de chuvas (STEINKE, 2012).

A sazonalidade pluviométrica do sudeste brasileiro concentra as chuvas no período do verão (DJF - Dezembro/Janeiro/Fevereiro). Os mecanismos responsáveis pelas chuvas nessa região mais evidentes, são os sistemas frontais (SF), as variações nos padrões de chuvas vinculados à ZCAS (Zona de Convergência do Atlântico Sul), bem como o processo urbanização acelerada, do uso do solo, e dos mecanismos dinâmicos e termodinâmicos (DUFEK; AMBRIZZI, 2008; VÁSQUEZ P. et al., 2018; ZILLI et al., 2017).

A convecção também é marcante no sudeste brasileiro e promove grandes valores acumulados de chuvas dependendo da velocidade do deslocamento dos SFs (TEIXEIRA; SATYAMURTY, 2011).

Além disso, a ocorrência da ZCAS, no período chuvoso, eleva a possibilidade de chuvas intensas, mesmo que não exclua a possibilidade de extremos de chuvas no período de estiagem ou de transição (CARVALHO; JONES; LIEBMANN, 2004; ZANDONADI et al., 2016). 
Tais sistemas atmosféricos se inserem sobre uma zona de atuação de massas de ar equatoriais, tropicais e polares. O período que compreende o verão é o dos máximos de precipitação que migram para o Norte do Equador durante o período do inverno, marcados pela estiagem (REBOITA et al., 2010, 2012).

O dinamismo do clima, portanto, pressupõe a observação das massas de ar, da pressão atmosférica, da temperatura do ar, da umidade relativa, da radiação solar, das chuvas, etc. Tais observações, de maneira geral, favorecem para a compreensão do clima regional, além de não excluir a necessidade de sua análise individual (SILVEIRA, 2008).

Para a compreensão da gênese das chuvas (pluviométrica), a obtenção de dados, por sua coleta e tabulação é tarefa difícil que, além de crucial, no Brasil não conta adequadamente com a compreensão de entidades que detém e gerenciam dados meteorológicos, uma vez que esses poderiam colaborar de forma ampla e irrestrita tais elementos (ZAVATTINI; BOIN, 2013).

Contudo, as dificuldades observadas na análise climática em diferentes regiões brasileiras são perceptíveis, como aponta Santos (2014), que na análise junto ao bioma do cerrado no Distrito Federal/Brasil, considera a precipitação com elevada variabilidade temporal e destaca para a necessidade de estudos que correlacionem à análise de variáveis climáticas com a urbanização e o uso da terra. Nessa região, o autor ressalta o aumento no número de dias consecutivos secos e o aumento nas chuvas fortes, além de se concentrar em poucos dias (máximo acumulado em 5 dias), sendo tais dados obtidos através de índices climáticos pelo RClimdex (ZHANG; YANG, 2004).

Já no Estado de São Paulo, notou-se que, apesar das tendências positivas (aumento) para alguns índices climáticos, como no total de chuvas acumulada anualmente ou no número de dias acima de $20 \mathrm{~mm}$ de chuva. Contudo destacam-se algumas contradições, como apontado na comparação entre o número máximo de dias consecutivos secos e os dias acima de $20 \mathrm{~mm}$ de precipitação, em que a significância negativa para existência de tendência contrasta com as chuvas intensas se concentrando em poucos dias (DUFEK; AMBRIZZI, 2008).

Uma característica interessante nesse sentido apontado por Santos et al. (2012) foram as relações com a produtividade agrícola, sendo as tendências positivas um fator de compensação junto às perdas por evapotranspiração dos organismos vegetais.

Segundo Haylock et al. (2006) os índices de máximo de dias consecutivos secos e úmidos, bem como o máximo acumulado de chuvas em um único dia e em cinco dias, expressam, com significativa relevância, um indicativo de acompanhamento diário e unificado sobre as mudanças nos extremos de chuva, enquanto índices para a observação de padrões anômalos individuais no clima, entendendo que a identificação de padrões de mudança na região sudeste da América do Sul, bem como a observação de padrões de circulação e da média anual de pressão atmosférica nos oceanos se faz necessária para o amplo entendimento da conjuntura estabelecida sobre a referida área. 
Atentando para isto, o presente trabalho buscou compreender especificamente o comportamento das chuvas na região de São Carlos/SP, a partir da análise dos dados disponibilizados por uma série histórica de 22 anos (1993-2014) recorrendo ao emprego de índices climáticos para as chuvas (ZHANG; YANG, 2004; HAYLOCK et al., 2006).

\section{Materiais e Métodos}

Foram utilizados os dados de chuvas diárias de três estações climatológicas da região de São Carlos, interior do Estado de São Paulo (Figura 01).

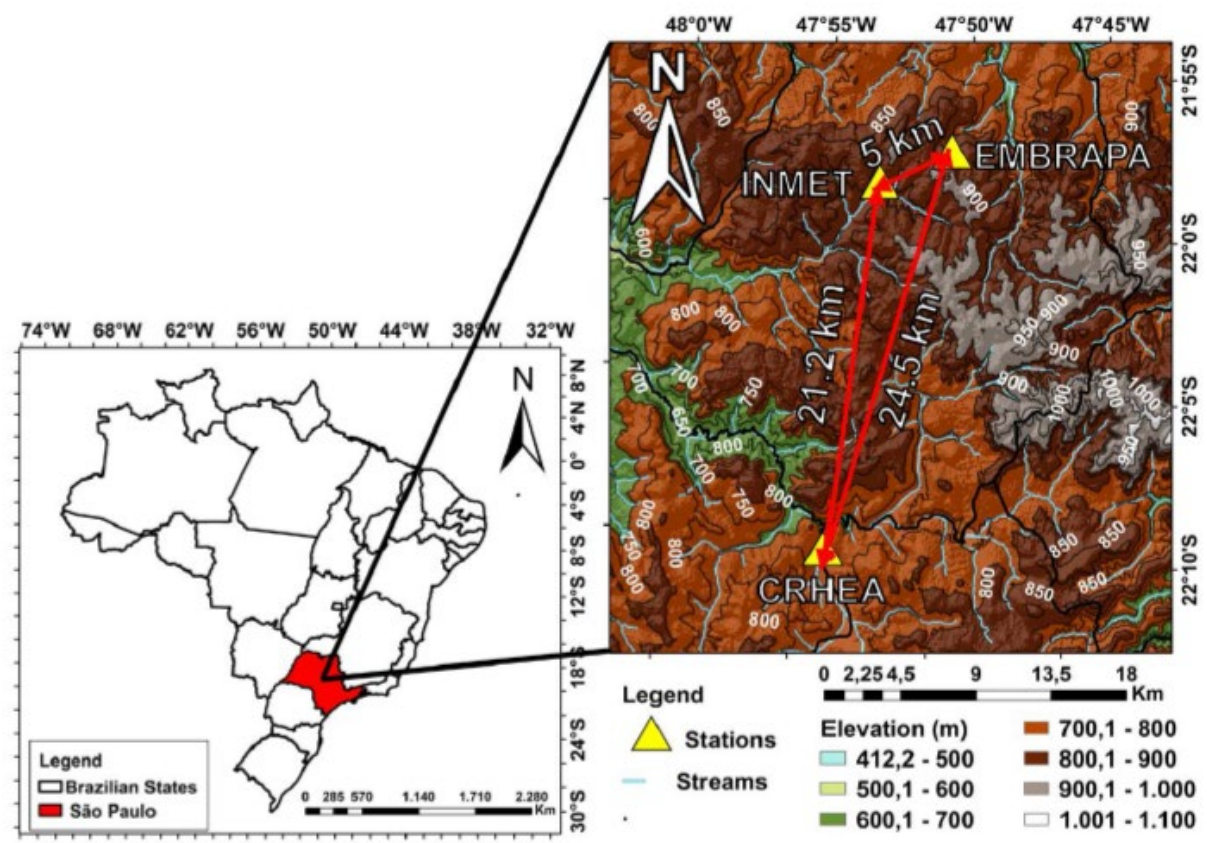

Figura 01: Localização das estacoes climatológicas.

Fonte: Adaptado de IBGE (2009)

De acordo com Monteiro (1973) a área e as estações climatológicas encontram-se estabelecidas sob os mesmos sistemas atmosféricos (Figura 02) sendo que, o período de análise de 22 anos de dados, se deu em função da existência de medições nos mesmos períodos entre as três estações analisadas, a fim de respeitar uma escala de abordagem espacial e temporal adequadas. 


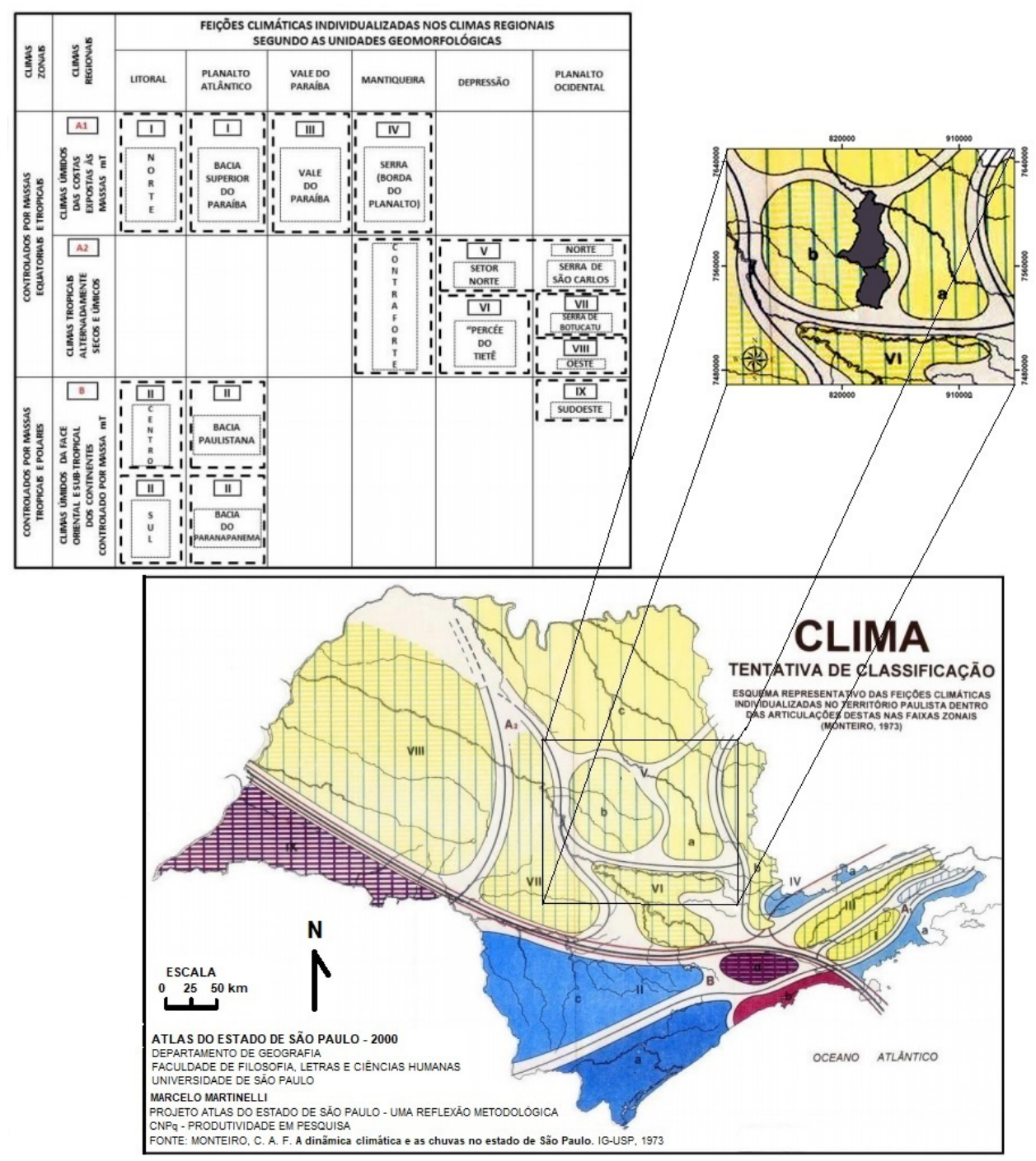

Figura 02: Classificação climática do Estado de São Paulo segundo Monteiro 1973, com as localidades dos municípios de São Carlos e Itirapina.

Fonte: Adaptado de Martinelli (2010).

As estações utilizadas foram respectivamente: a) INMET/UFSCar (Instituto Nacional de Meteorologia/Universidade Federal de São Carlos), b) EMBRAPA/Pecuária 
Sudeste (Empresa Brasileira de Pesquisa Agropecuária) e, c) CRHEA/EESC/USP (Centro de Recursos Hídricos e Estudos Ambientais/Escola de Engenharia de São Carlos/Universidade de São Paulo).

Os índices calculados e utilizados, acerca das chuvas diárias, foram: A) RX 1 dia; B) SDII; C) DCU; D) DCS; E) PRCPOT, conforme descrito na Tabela 01. Os índices se baseiam na metodologia de Zhang e Yang (2004) e Haylock et al. (2006), e foram calculados no ambiente RClimdex.

Tabela 01. Descrição dos índices climáticos utilizados nesse estudo

\begin{tabular}{c|c|c|c}
\hline Índices & $\begin{array}{c}\text { Nome dos } \\
\text { índices }\end{array}$ & Definição dos índices & Unidades \\
\hline \multirow{2}{*}{ RX1day } & $\begin{array}{c}\text { Máximo de } \\
\text { chuva } \\
\text { acumulado } \\
\text { em 1 dia }\end{array}$ & $\begin{array}{c}\text { Máximo de chuva acumulada em um } \\
\text { único dia }\end{array}$ & mm \\
\hline \multirow{2}{*}{ SDII } & $\begin{array}{c}\text { Índice } \\
\text { simples de } \\
\text { intensidade } \\
\text { diária }\end{array}$ & $\begin{array}{c}\text { Total anual de chuvas acumulado } \\
\text { dividido pelo número de dias úmidos } \\
\text { (quando o dia esteve }>=1.0 \mathrm{~mm} \text { ) no ano }\end{array}$ & mm/dia \\
\hline \multirow{2}{*}{ DCS } & $\begin{array}{c}\text { Dias } \\
\text { consecutiv }\end{array}$ & $\begin{array}{c}\text { Número máximo de dias em que as } \\
\text { chuvas }>=1 \text { mm }\end{array}$ & dias \\
& $\begin{array}{c}\text { Dias } \\
\text { consecutiv } \\
\text { os secos }\end{array}$ & $\begin{array}{c}\text { Número máximo de dias em que as } \\
\text { chuvas }<1 \text { mm }\end{array}$ & dias \\
\hline PRCPTOT & $\begin{array}{c}\text { Total de } \\
\text { chuvas } \\
\text { acumulada } \\
\text { s em um } \\
\text { ano }\end{array}$ & $\begin{array}{c}\text { Valor total anual de chuvas acumuladas } \\
\text { nos dias úmidos (dias }>=1 \text { mm) }\end{array}$ & mm \\
\hline
\end{tabular}

Fonte: Adaptado de Zhang e Yang (2004).

O script foi desenvolvido por Byron Gleason do National Climate Data Center (NCDC) da National Oceanic and Atmospheric Administration (NOAA), cujo uso é apresentado em diversos workshops e reuniões do CCI/CLIVAR (International Research Programme on Climate Variability and Predictability) sobre elementos climáticos e sua variabilidade desde 2001 (HAYLOCK et al., 2006; SANTOS et al., 2012).

Os índices são calculados junto ao software R, que é um ambiente e uma linguagem para computação estatística e gráfica, sendo esse um software livre (Projeto GNU) similar à 
linguagem e ao ambiente $\mathrm{S}$, desenvolvido nos laboratórios Bell (antigo AT \& $\mathrm{T}$, agora Lucent Technologies).

O R apresenta diversas modelagens lineares e não lineares, testes estatísticos clássicos, análise de séries temporais, classificação, clustering, entre outras técnicas estatísticas e gráficas, além de fornecer uma rota open source para participação nessa atividade.

Diversos trabalhos utilizaram a ferramenta a fim de analisar a evolução temporal e espacial dos climas em diferentes regiões do Brasil e de outros países (DRUMOND; AMBRIZZI, 2008; DUFEK; AMBRIZZI, 2008; HAYLOCK et al., 2006; MEKIS; VINCENT, 2011; SANTOS et al., 2012; SANTOS; RAMOS, 2018; STEPHENSON et al., 2014).

O RClimdex calcula 27 índices básicos recomendados pela ETCCDMI (Climate Change Detection Monitoring Indices) para temperatura e precipitação (KARL; NICHOLLS; GHAZI, 1999); PETERSON, et al., 2001), dos quais foram utilizados, apenas, os índices associados à precipitação pluviométrica, conforme descrito na tabela 2 (HAYLOCK et al., 2006; SANCHES, 2015; SANTOS et al., 2012; STEPHENSON et al., 2014; ZHANG; YANG, 2004).

O controle de qualidade dos dados se fez pela substituição de todos os dados faltosos por -99.9 (reconhecido como faltoso pelo script).

Os resultados dos índices calculados geram um arquivo de texto sem formatação, que foram organizados, separadamente, em planilhas individuais para cada índice (SANCHES, 2015; SANTOS et al., 2012).

Estes valores incluem as quantidades de precipitação diárias menores que zero e, as estações climatológicas utilizadas, onde foram apresentadas falhas de dados em alguns dos anos em estudo, sendo mais crítico o caso da estação INMET/UFSCar, onde os dados faltosos no ano de 1999 superaram 5\%, enquanto as estações CRHEA/EESC/USP e EMBRAPA não apresentaram falhas significativas.

A partir dos índices calculados foi possível compreender o comportamento pluviométrico das chuvas a partir do máximo acumulado em um único dia, a intensidade pluviométrica diária, eventos extremos de precipitação, dias consecutivos secos, dias consecutivos úmidos e a precipitação anual nos dias para a série histórica em estudo.

Os índices foram analisados a partir da construção de gráficos com suas respectivas variáveis para as três estações climatológicas observadas, além disso, foi atribuído para cada um dos índices, o valor médio de toda a série histórica para comparação entre períodos acima ou abaixo desse em meio às flutuações interanuais dos dados.

As representações dos índices calculados influem na análise do comportamento das chuvas a partir de dados históricos e, pelo fato de interpretar dados diários, o uso do software corrobora na melhor compreensão de fenômenos climatológicos, além de minimizar o uso, apenas, das médias climatológicas, que podem ocultar as realidades do clima em diferentes 
escalas por desprezar as sequências de dias chuvosos, da intensidade das chuvas, entre outros. Contudo, esse apresenta algumas limitações quanto à maleabilidade e adaptação ferramental para a realidade climática tropical, uma vez que a distribuição sazonal das chuvas existentes nessas regiões apresenta ritmos de flutuações interanuais.

\section{Resultados e Discussão}

A partir dos resultados obtidos, notaram-se períodos em que os valores estiveram acima ou abaixo dos valores médios estabelecidos para cada índice. Tais flutuações são atribuídas à influência ou à ausência de fenômenos climáticos responsáveis pela gênese pluviométrica regional mencionadas anteriormente.

Um estudo realizado na região de São Carlos/SP apontou nos anos caracterizados como secos, ou seja, com volumes pluviométricos abaixo dos valores médios (como nos anos de 2013 e 2014), verificou-se a ausência de eventos de ZCAS que se manifestam principalmente no período de verão para a região, o que corroborou para a redução do volume pluviométrico precipitado (SANTOS et al., 2017).

Sendo assim, a ocorrências da ZCAS é fundamental para a análise dos volumes pluviométricos na área de estudo, pois é responsável pelos elevados registros de chuvas diárias e consecutivas.

A Zona de Convergência do Atlântico Sul transporta umidade para a área de estudo, sobretudo entre os meses de outubro a março (primavera-verão), mediante a articulação dos sistemas dirigidos pela mEc (Massa Equatorial Continental) e mTa (Massa Tropical Atlântica) que atuam respectivamente sobre a porção oeste da região Amazônica e porção sudeste do oceano Atlântico abaixo da linha do Equador; portanto, seu bloqueio ou manifestação repercute sobre o índice pluviométrico da área de estudo ocasionando estiagens ou aguaceiros.

Além disso, a presença nesse período (2013 e 2014) de um sistema de alta pressão orientado mais a oeste do Atlântico Sul nas imediações do Trópico de Capricórnio, fenômeno denominado ASAS (Alta Subtropical do Atlântico Sul), estabeleceu um bloqueio atmosférico que impediu o avanço de frentes frias ou a manifestação habitual da ZCAS, privando a área da conjuntura que favorece o fluxo de umidade que a abastece (COELHO et al., 2016).

Vale ressaltar ainda, que os sistemas frontogenéticos, a ZCAS e seus fenômenos correlatos, concentram os principais fatores responsáveis pelas chuvas na região.

A dinâmica climática regional também se associa à interação atmosfera-oceanocontinente, que expressam as teleconexões e suas influências na variabilidade climática. Tais teleconexões subsidiam a compreensão da flutuação climática por meio de dados oceânicos do Atlântico e do Pacífico, como a OMA (Oscilação Multidecadal do Atlântico), as fases do El Niño - sua fase positiva (ENSO - El Niño Oscilação Sul; aumento na temperatura do 
Pacífico) e La Niña - sua fase negativa (resfriamento na temperatura do Pacífico), a ODP (Oscilação Decadal do Pacífico), entre outros.

As teleconexões e sua influência na dinâmica climática regional para o sudeste do Brasil podem ser observados em diferentes trabalhos que constataram sua correlação com as tendências de aumento/diminuição das temperaturas e precipitação nos períodos e localidades analisadas (DRUMOND; AMBRIZZI, 2008; EVANGELISTA et al., 2007; KAYANO; CAPISTRANO, 2014; PSCHEIDT; GRIMM, 2009; VÁSQUEZ P. et al., 2018).

Atentando para isto, a análise dos dados no ambiente RClimdex, demonstrou que o RX de 1 dia (Figura 04) da série alcançou valor médio de 79,8 $\mathrm{mm}$ de chuva entre as estações, destacando que os anos de 1997, 2008 e 2014 mantiveram-se em todas elas distantes do valor médio esperado do máximo de precipitação pluviométrica em um único dia. Não obstante a isto, em todos os anos obteve-se ao menos $45 \mathrm{~mm}$ de chuva num único dia sendo que os mesmos apresentaram-se no período do semestre hidrológico que se estende de outubro a março.

Em $81 \%$ dos anos analisados esses valores se encontraram próximos ao esperado. Além disso, a estação climatológica do CRHEA/USP foi a que apresentou os maiores valores extremos diários de chuva que se situaram nos anos de 2003, 2011 e 2013, com 128, 158 e $151 \mathrm{~mm}$ de chuvas, respectivamente.

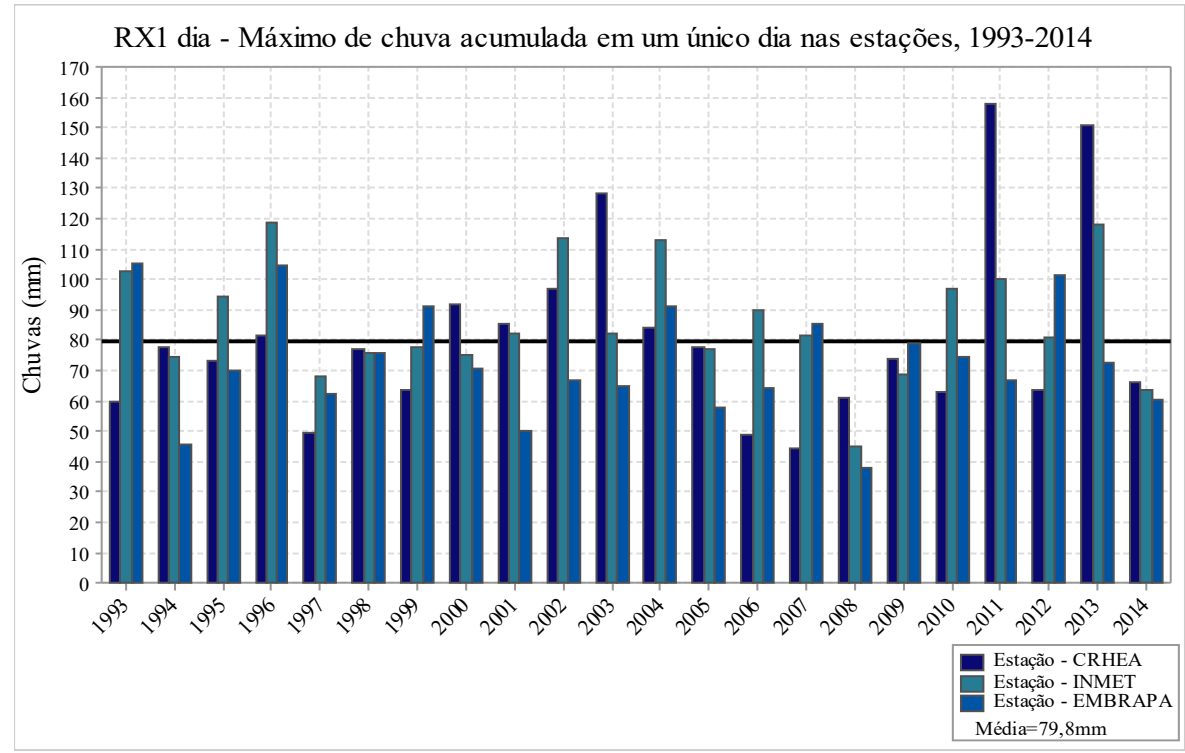

Figura 04: RX 1 dia - Valores máximos de chuva acumulada em um único dia e valor médio referencial nas estações, 1993-2014. 
Diante das médias obtidas $(79,8$ e $45 \mathrm{~mm})$ com a aplicação da metodologia, é possível prever a ocorrência de dias chuvosos em todos os anos nos quais a precipitação alcance ao menos $45 \mathrm{~mm}$ num intervalo de 24 horas, o que pode impactar significativamente tanto o espaço rural como o urbano a depender da intensidade do evento.

Pelos resultados obtidos pode-se notar que as chuvas que se manifestam com as características delineadas pelos valores máximos acumulados em um dia (RX 1 dia) sempre ocorreram no semestre hidrológico que vai de outubro a março, assumindo na área de estudo a condição sazonal que se estabelece a partir da estiagem que se verifica de abril a setembro. Também é importante ressaltar que as chuvas desse período úmido se concentram nos meses de dezembro, janeiro e fevereiro mesmo que os índices registrados em outubro, novembro e março sejam significativos e bem acima daqueles anotados no semestre seco.

Mesmo com a observação das ocorrências sazonais, nota-se a importância dos índices climáticos em longas séries de dados, uma vez que a predição ou a análise do comportamento dessas pode direcionar o planejamento e, até mesmo, a tomada de decisão em plantios agrícolas, ações preventivas no espaço urbano, ou ainda programação de afazeres ao longo do ano entre outros (SANCHES et al., 2017, 2018).

O índice SDII (Figura 05) representa a intensidade média de chuvas anuais a partir da série histórica adotada. Através de sua obtenção pode-se observar o comportamento do valor acumulado de chuva diária ao longo dos anos. A série de dados apresentou uma lacuna no ano de 1999 para a estação do INMET/UFSCar.

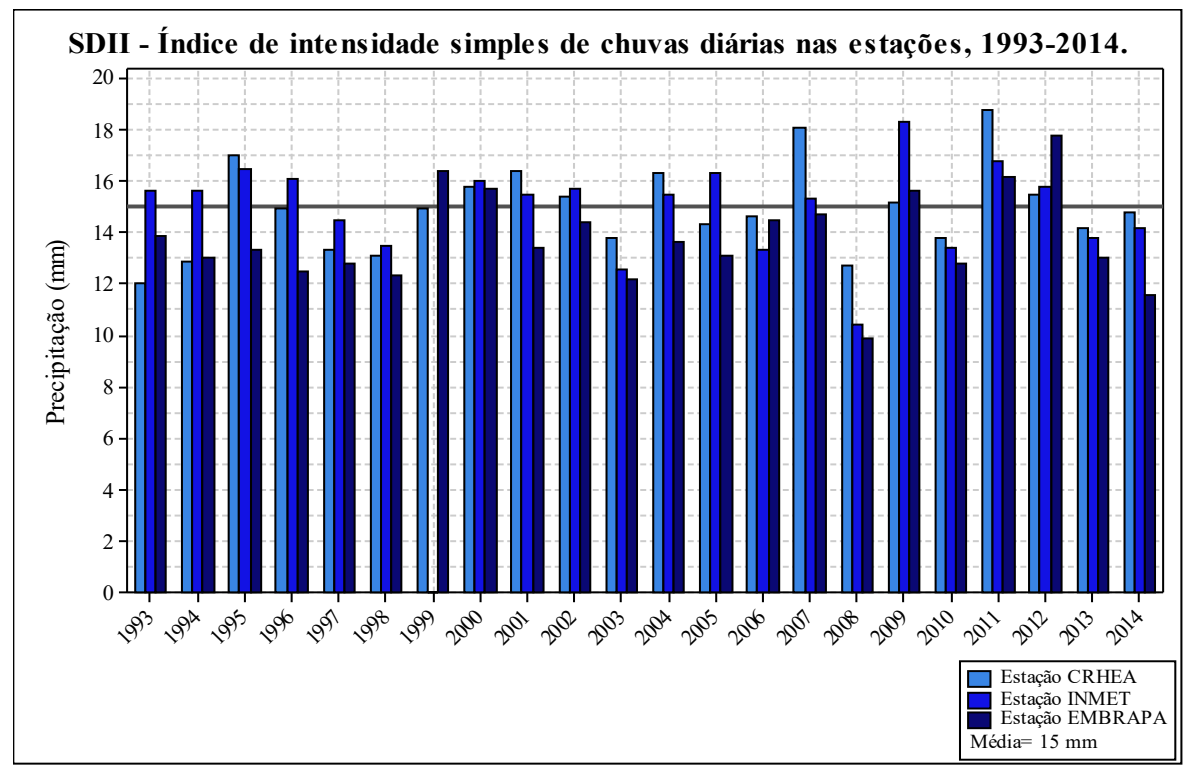

Figura 05: SDII - Intensidade simples das chuvas e valor médio referencial nas estações entre 1993-2014. 
Conforme é possível observar, o valor médio de chuva obtido para o SDII foi de 15 mm, sendo que em 2000, 2009, 2010 e 2011 os índices analisados encontram-se acima da referenciada média para todas as estações enquanto que em 1997, 1998, 2003, 2006, 2008, 2010, 2013 e 2014 a intensidade de chuva ficou abaixo da média obtida, alcançando o dobro de anos em que os valores anotados foram superiores à média, destacando-se na perda o ano de 2008 e no ganho 2011.

A interpretação dos valores acumulados de chuvas, auxiliada pelo cálculo do SDII que possibilita o delineamento da quantidade de precipitação por sua intensidade, em seu comportamento anual, permitiu verificar que não ocorreram mudanças no regime das chuvas com base nesse índice, uma vez que a relação entre a quantidade de chuvas e os dias chuvosos não apresentaram variações de intensidade muito distantes do esperado com base no valor médio admitido para o período, exceto no ano 2008 que apresentou em todas as estações valor médio situado abaixo de $12 \mathrm{~mm}$ de chuvas nos dias chuvosos, diferindo dos demais anos que apresentaram médias acima dos $12 \mathrm{~mm}$ de chuvas. Diante desses valores de intensidade simples de chuvas e sua distribuição anual, pode-se estabelecer uma estimativa básica do volume de chuvas esperadas em um dia chuvoso, na região analisada.

A disposição dos dias consecutivos úmidos e secos é uma das mais significativas preocupações quanto à dinâmica e o ritmo das chuvas na região estudada, uma vez que nessas áreas, as flutuações nos volumes de chuvas e sua distribuição temporal (diária, mensal, anual, etc.) apresentam-se complexas e, a partir daí, correspondem à concentração ou dispersão dos dias consecutivos chuvosos.

O cálculo dos índices DCU e DCS (Figuras 06 e 07) informa a expectativa de frequência dos dias úmidos e secos consecutivos, ainda que não estabeleça com exatidão o período específico (dias, meses e semestres) de sua ocorrência ao longo do ano, no entanto, permite o estabelecimento de um parâmetro anual, assinalando os valores máximos de dias consecutivos úmidos ou secos anuais que podem ocorrer na área de estudo.

Graças as análises desenvolvidas, verificou-se que os DCU se manifestam, no período mínimo de 5 dias consecutivos chuvosos na série histórica, o que pode ser associado aos eventos da ZCAS, um evento característico do período chuvoso (primavera-verão) no Sudeste brasileiro e que implica na concentração do número de dias consecutivos chuvosos em sua variabilidade interanual (QUADRO et al., 2012; CARVALHO et al., 2004; ÁVILA et al., 2016). O cálculo desses índices não considera a quantidade de dias consecutivos úmidos e secos que não correspondem ao máximo de dias em um único ano, ou seja, apenas mensura, anualmente, quais os valores máximos dos dias consecutivos secos e úmidos, o que representa uma lacuna junto ao processo interpretativo do índice, mesmo que esse considere os dados climatológicos diários para o cálculo. 


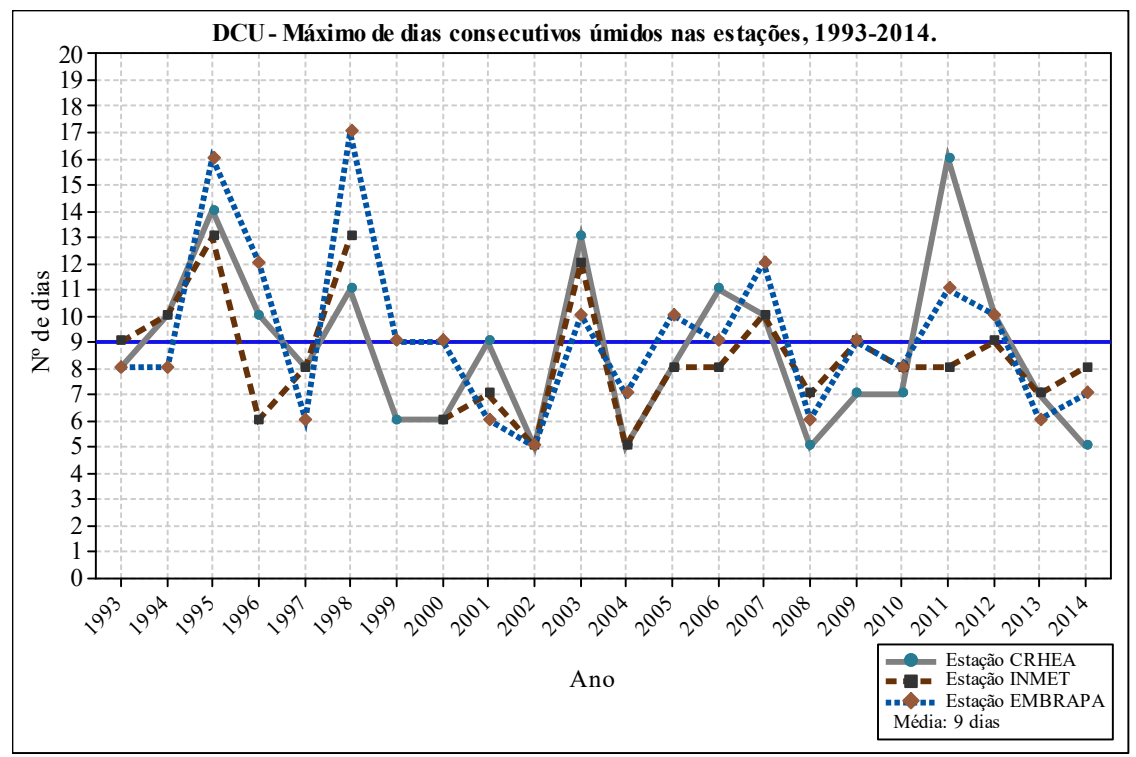

Figura 06: DCU - Valores máximos de dias consecutivos úmidos e referencial médio nas estações, 1993-2014.

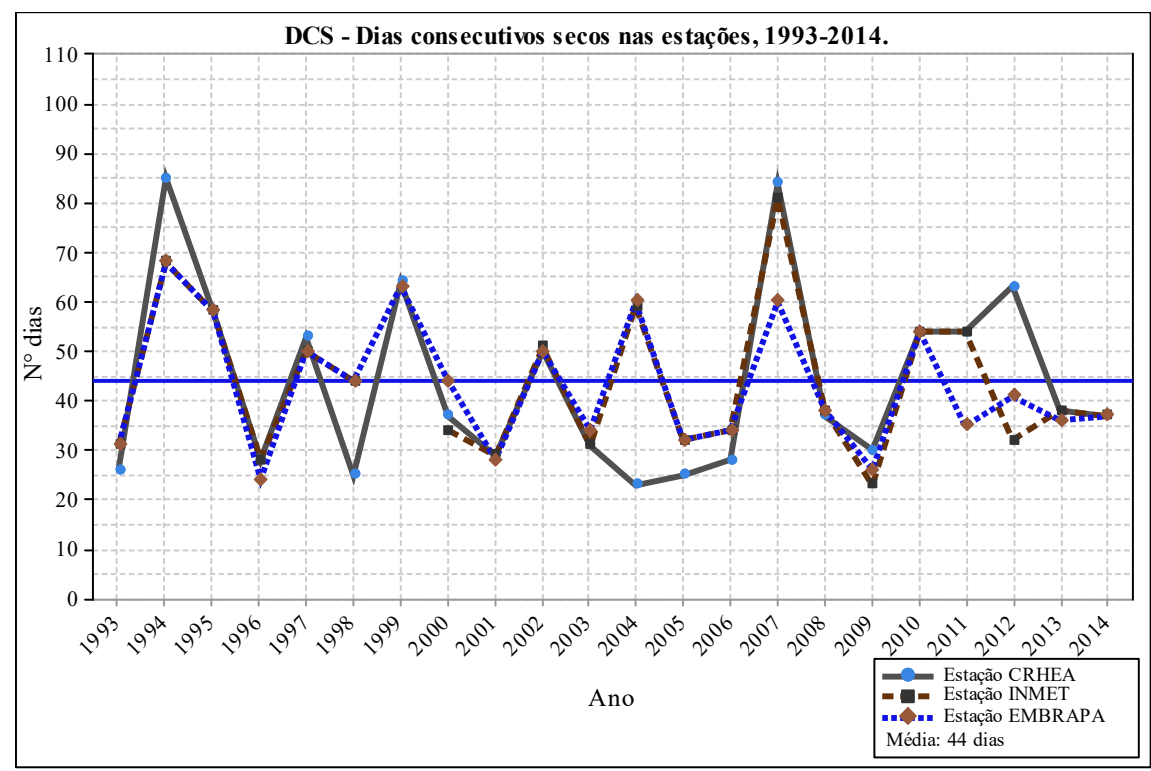

Figura 07: DCS - Valores máximos de dias consecutivos secos e referencial médio nas estações entre 1993-2014. 
Ainda assim, os índices de DCU e DCS demonstram a concentração do máximo de dias consecutivos chuvosos e secos, que são possíveis e identificáveis por inferência dos dados obtidos, permitindo identificar flutuações marcantes entre os anos da série histórica, enquanto o comportamento entre as estações climatológicas apresentaram-se semelhantes, exceto nos poucos anos em que uma das estações apresentou valores extremos, como registrado no ano de 2011 na estação localizada no CRHEA/USP em relação às outras para os dias consecutivos úmidos.

Esses índices permitem interpretar a presença de inúmeros dias sem chuvas consecutivos, que ocorrem, predominantemente, no inverno (entre os meses de julho e agosto principalmente), enquanto os valores de dias consecutivos úmidos se concentram no período do semestre hidrológico, mesmo que a análise de sua concentração e/ou dispersão seja inviabilizada pelos resultados obtidos a partir do seu cálculo.

A identificação de DCUs mediante a análise dos dados das estações climatológicas indica a presença de flutuação no período considerado, uma vez que são verificados anos abaixo e acima da média de máximo de dias consecutivos úmidos, algo situado na conjuntura da área, pois, ao menos nove dias consecutivos úmidos são anotados em todos os anos da série histórica. Outro aspecto referente aos dados, diz respeito a existência de anos que se encontram com valores acima do esperado junto aos dias consecutivos úmidos, como é observado nos anos de 1995, 1998 e 2011.

Em face da média de 9 dias obtida para os anos, observa-se nos últimos três anos da série histórica um decréscimo no máximo de dias consecutivos úmidos principalmente na estação do CRHEA/USP, enquanto as outras estações apresentam um pequeno aumento.

No período, cerca de $54 \%$ dos anos apresentaram-se com máximo de dias consecutivos úmidos abaixo do esperado (1993, 1997, 2000, 2001, 2002, 2004, 2005, 2008, $2009,2010,2013$ e 2014), enquanto quase $23 \%$ se encontram significativamente acima da média anual esperada (1995, 1998, 2003, 2007 e 2012).

Nos anos de 1994 e 2007 o máximo de dias consecutivos secos chegou a oitenta dias, o que representa mais de dois meses sem chuvas no período de um ano. Já nos anos de 2004 e 2009, pode-se observar que o máximo de dias sem chuva não ultrapassou o total de vinte e cinco.

Apesar dos índices excepcionais encontrados na série histórica, nota-se também uma grande flutuação nos dias consecutivos secos no período dos últimos quatro anos, sendo que, como existem grandes oscilações interanuais, as observações que se seguem em toda a série histórica não apontam para um padrão nesse aspecto, mesmo quando são verificadas diferenças nos dados das estações climatológicas.

A média dos dias consecutivos secos para as estações climatológicas é de quarenta e dois, sendo que as estações registraram ao menos dez anos em que os dias consecutivos secos estiveram acima da média e pelo menos sete anos que estiveram abaixo da média esperada para o período. Nos últimos quatro anos, nota-se que esses estiveram na média 
esperada ou acima para período na série histórica adotada, em função da média calculada para os dados analisados.

$\mathrm{O}$ índice de PRCPTOT (Figura 08) refere-se à quantidade total de chuvas nos dias úmidos, sendo esses os dias em que se registrou a menor incidência de chuvas por milímetro. A partir desse índice pode-se observar a quantidade total de chuvas, anualmente, para série histórica adotada (1993-2014) nas três estações climatológicas. No presente estudo, foi admitido como valor médio base de PRCPTOT $1538 \mathrm{~mm}$, uma vez que representa a média anual das chuvas na série histórica entre as estações climatológicas que forneceram os dados para análise.

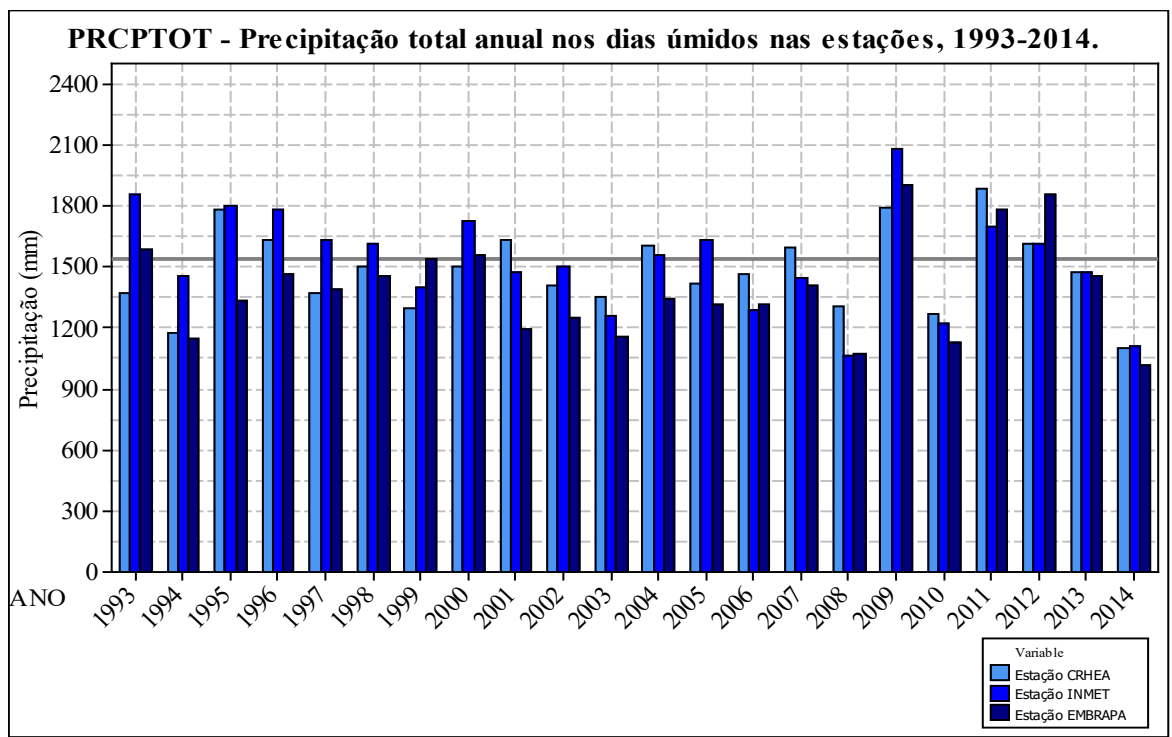

Figura 08: PRCPTOT - Total acumulado de chuvas e valor médio referencial nas estações entre 1993-2014.

As flutuações nos dados se associa também, à influência das teleconexões. Tais flutuações ocorreram em um período considerado de baixa variabilidade nos volumes pluviométricos quando comparados a outras normais climatológicas (longas séries de dados pluviométricos). Através dos dados é possível verificar a influência das teleconexões, mesmo que de forma sutil, como se observa no período de 1998-2000 onde a normalidade dos índices registrados da PRCPTOT e SDII frente a série histórica, manifesta coincidência com a ocorrência de La Niña (tendência negativa para chuvas), que favorece a redução dos DCU e o aumento dos DCS, por cooperar com a concentração das chuvas em menos dias durante o ano. Já no período de 2006-2012, que apresenta uma fase de tendência positiva para chuvas (incremento no volume pluviométrico interanual), notou-se o aumento dos volumes de chuvas em PRCPTOT, SDII, RX1 e 5 dias, especialmente no período 2009- 
2012, onde se observou um período negativo para El Niño e para a ODP, porém, positivo para a AMO, evidenciando o aumento na pluviosidade (VÁSQUEZ et al., 2018).

Outro período de destaque é o biênio 2013-2014, em que se observa a redução em todos os valores pluviométricos na série histórica observada, além da redução dos DCU e dos DCS, coincidindo com um período de neutralidade de El Niño (ENSO). Contudo, como a tendência para as chuvas foi negativa, observa-se sua associação com a mudança da ODP de uma fase negativa para uma fase positiva que pode reafirmar a redução dos valores pluviométricos (BARREIRO; TIPPMANN, 2008; CARVALHO; JONES; LIEBMANN, 2004; RAO et al., 2016; RAO; HADA, 1990; VÁSQUEZ et al., IDEM).

A partir do cálculo das chuvas totais nas estações, observou-se que apenas em três anos da série histórica $(2009,2011$ e 2012) todas as estações climatológicas apresentaramse acima do valor referencial médio de $1538 \mathrm{~mm} \mathrm{e}$, em oito do total de vinte e um anos, encontraram-se abaixo desse mesmo valor (1994, 2002, 2003, 2006, 2008, 2010, 2013 e 2014).

Outra característica presente nos dados é a presença de flutuações anuais nos valores de chuvas, e até mesmo entre as estações climatológicas, registrando que em treze dos vinte e um anos da série pelo menos uma delas apresentou chuvas acima do valor médio obtido.

Nos anos últimos seis anos, as estações climatológicas apresentaram três anos (2009, 2011 e 2012) com valores de chuvas anuais acima do referencial médio de $1538 \mathrm{~mm}$, e outros três anos (2010, 2013 e 2014) em que as chuvas estiveram abaixo do valor médio adotado, o que expressa a flutuação presente nos dados na série histórica a partir do total acumulado da precipitação com, ao menos, 1 milímetro de chuva em relação aos dados diários.

O curso e distribuição das chuvas totais acumuladas em um ano (PRCPTOT), por exemplo, reafirmaram as flutuações interanuais das chuvas, apresentando volumes acima do índice médio em determinados anos, assim como valores abaixo do esperado para outros anos no mesmo período anual. Obviamente isso manifesta-se mesmo que não a variabilidade não se manifeste entre os meses ou período considerado como semestre hidrológico no qual as chuvas se concentram durante o ano devido as características atmosféricas/climatológicas da região estudada. Ainda em relação aos totais anuais acumulados de chuva, pode-se notar que a média esperada para um ano na região encontrou-se próximo aos valores determinados pela ANA (2013), referentes à bacia hidrográfica em que São Carlos/SP está inserida, o que colaborou para a identificação de períodos de maior ou menor concentração de chuvas, e pode ser utilizado como ferramenta de planejamento em atividades agrícolas e/ou em atividades urbanas a fim de facilitar a compreensão da variação que as chuvas apresentam, e, sobre os impactos que ocasionam sobre essas áreas.

Outro aspecto relevante no comportamento pluviométrico regional é a paisagem onde essa se insere, uma vez que, em função da dinâmica atmosférica, essa região apresenta influência da umidade advinda da Massa de ar Tropical Atlântica, favorecendo eventos de 
chuvas em dias consecutivos e secos, bem como nos valores sazonais das chuvas para essa área (ZANDONADI et al., 2016).

Por fim, a limitação da série de dados dificulta o apontamento de análises de tendência para o regime pluviométrico, bem como não limita as discussões a respeito das flutuações sazonais nas chuvas para a região de estudo. Com isso, a ampliação das escalas temporal e espacial poderão colaborar para uma melhor observação das precipitações para São Carlos/SP e a região.

\section{Conclusões}

As considerações a respeito do comportamento da precipitação na região tropical guardam relativa complexidade que se somam ao entendimento das alterações em curso no regime de chuvas da área de estudo, pois, esses territórios constituem espaços onde a variabilidade atmosférica é recorrente, e, a compreensão de sua natureza dinâmica é dificultada pelas incertezas observadas nos dados climatológicos disponíveis e nos efeitos atmosféricos dinâmicos que se apresentam através dos fenômenos naturais.

Sob essa conjuntura, o estudo desenvolvido na região de São Carlos/SP demonstrou ao longo de sua redação como a análise de dados e a aplicação de metodologias ficam prejudicadas quando a fonte das informações necessárias à pesquisa apresenta falhas no tocante a qualidade dos dados disponibilizados ou ausência dos mesmos em alguns hiatos de tempo, pois o manuseio de dados confiáveis e constantes é condição sine qua non para o desenvolvimento de qualquer estudo independentemente da complexidade que possui.

Nessa pesquisa, a lacuna de dados, por exemplo, dificultou em alguns momentos o cálculo de alguns índices propostos (SDII, DCU e DCS) através da metodologia adotada que recorreu aos recursos analíticos proporcionados pelo programa RClimdex.

Verificou-se, ainda, que além da qualidade dos dados, existem diferenças admissíveis entre as estações climatológicas em razão da localização que possuem, pois, mesmo não sendo distantes espacialmente e observando as normatizações de instalação estabelecidas pela OMM - Organização Meteorológica Mundial, apresentam informações distintas em função, dentre outros da altimetria e do relevo (por seu efeito orográfico) de seu sítio.

Vale ressaltar, que as estações localizadas nos domínios do Planalto Ocidental Paulista em São Carlos/SP (INMET/UFSCar e EMBRAPA) apresentaram dados congruentes entre si na maior parte dos casos, contudo, a estação CRHEA/USP apresentou resultados ligeiramente diferentes das outras por situar-se na transição da Depressão Periférica Paulista para o Planalto Ocidental Paulista nos domínios das Cuestas Basálticas, onde o efeito orográfico ocasiona as condições diferenciadas das demais devido a ascensão das massas de ar e expansão adiabática resultante.

A PRCPTOT nas estações climatológicas apresentou grande flutuação interanual, sendo que, em apenas oito anos (36\% do total), os valores obtidos estiveram abaixo da média 
esperada de chuvas anuais para a região da Bacia do Paraná e para a região analisada, que se situa ao redor de $1500 \mathrm{~mm}$ (ZANDONADI, 2013; SANCHES, 2015).

Os DCUs e DCSs mostraram-se insuficientes para a compreensão da dispersão ou concentração das chuvas ao longo da série histórica, uma vez que informaram apenas o valor máximo de ocorrência para o cálculo do índice. Registra-se que o máximo de DCUs e DCSs oscilou em sintonia com o quadro geral, pois, quando os anos apresentaram maior volume anual de chuvas, seguiu-se a tendência de aumento no número máximo de DCUs, assim como foi manifesta a tendência de aumento no número máximo de DCSs quando os anos apresentaram menor volume de chuvas frente a média da série histórica.

Diante disto, observou-se que na região de São Carlos/SP e, provavelmente em todo o Sudeste brasileiro, a associação entre os DCUs e os eventos de ZCAS é significativa, pois, sua menor ocorrência nos últimos anos da série (2013 e 2014) fizeram-se sentir nos índices abaixo da média registrados, ocasionando diversas dificuldades para a sociedade e gestores públicos devido a diminuição da oferta de recursos hídricos no Estado de São Paulo e consequente racionamento de água implementado.

Os valores de RX 1 dia encontraram-se flutuantes na série histórica, contudo, os valores máximos de chuva acumulada em um único dia responderam pelos dias mais chuvosos nas estações climatológicas ao longo da série histórica.

Tais manifestações ocorreram ao longo dos meses de outubro, novembro, dezembro, janeiro, fevereiro e março em razão dos mesmos comporem o semestre hidrológico da área devido a concentração da precipitação que é favorecida pela ocorrência frequente tanto da ZCAS como dos processos frontogenéticos dentre outros.

Em razão disso, em toda a série histórica, ao menos um dia apresentou mais de 40 mm de chuva em alguns desses meses, favorecendo um melhor entendimento de sua gênese a partir dos processos supracitados no parágrafo anterior que atuam na região, bem como de sua repercussão espacial e temporal, ocasionada pelos aguaceiros de intensidade elevada que provocam enchentes e inundações que ocasionam, dentre outros, perda de plantios na zona rural e danos materiais nas áreas urbanas quando as águas arrastam bens, danificam edificações e infraestrutura ou ainda invadem casas e estabelecimentos comerciais comprometendo seus bens e produtos (SANCHES et al., 2018)

Além disso, os valores de SDII que descrevem a intensidade (simples) das chuvas nas estações climatológicas, também apresentaram similaridade com os anos de maior volume de precipitação anual. Com isso, a intensidade pluviométrica em dias chuvosos alcançou ao menos $10 \mathrm{~mm}$ em todos os anos calculados, sendo que o valor médio de intensidade das chuvas nas estações analisadas foi de $15 \mathrm{~mm}$ para a região de São Carlos/SP.

Diante dessas avaliações e resultados, constatou-se que uma série de dados dessa magnitude não é suficiente para o entendimento pleno e conclusivo do regime pluviométrico regional, entretanto, permite que outras informações sejam obtidas para nortear o planejamento dos agentes públicos frente aos desafios que se apresentam em eventos de 
estiagem prolongada ou na ocorrência de chuvas intensas e extremas na região (SANTOS et al., 2017; SANCHES et al., 2018).

Nesse aspecto, são planejados e estimulados novos estudos aplicando a metodologia utilizada na presente pesquisa em outras porções do território nacional e na presente área de estudo, ampliando a escala temporal e o número de estações a fim de expandir a base de dados analisados e aprofundar o embasamento que fundamenta a identificação dos padrões, alterações e tendências que norteiam o regime de chuvas nas mesmas, pois, tal elenco de informações proporcionará os elementos fundamentais que auxiliam na gestão dos recursos hídricos com base no comportamento anual da precipitação, oferta hídrica, demanda permanente da sociedade e parâmetros ambientais.

Para os centros urbanos e áreas rurais, uma projeção dos eventos com base no volume precipitado e intensidade (duração), auxiliará no redimensionamento dos sistemas de drenagem e na captação do escoamento superficial, evitando inundações e prejuízos, proporcionando reserva e suprimento para o período de estiagem, minimizando ou até mesmo erradicando tanto o racionamento que suspende as atividades econômicas e priva a população de abastecimento, bem como o comprometimento dos rios e lagos pela retirada excessiva de água de seus leitos.

Enfim, o horizonte dessa abordagem metodológica pode, inclusive, contribuir com o conjunto de estratégias admitidas e necessárias à gestão das Usinas Hidroelétricas em razão da relação que existe entre o volume de água armazenado nos reservatórios e a geração de energia, expandindo sua importância, aplicação e abrangência.

\section{Referências Bibliográficas}

ANA. Conjuntura dos recursos hídricos no Brasil: 2013. Agência Nacional de Águas. Brasília: ANA, 2013.

ÁVILA, A.; JUSTINO, F.; WILSON, A.; BROMWICH, D.; AMORIM, M. Recent precipitation trends, flash floods and landslides in southern Brazil. Environmental Research Letters, v. 11, n. 11, p. 114029, 2016.

BARREIRO, M.; TIPPMANN, A. Atlantic modulation of El Niño influence on summertime rainfall over southeastern South America. Geophysical Research Letters, v. 35, n. 16, 21 ago. 2008.

CARVAlHO, L. M. V.; JONES, C.; LIEBMANN, B. The South Atlantic Convergence Zone: Intensity, Form, Persistence, and Relationships with Intraseasonal to Interannual Activity and Extreme Rainfall. Journal of Climate, v. 17, n. 1, p. 88-108, 1 jan. 2004.

COELHO, C. A. S.; CARDOSO, D. H. F.; FIRPO, M. A. F. A seca de 2013 a 2015 na região sudeste do Brasil. Climanálise - Edição Especial de 30 anos, p. 55-61, 2016. 
DRUMOND, A. R. DE M.; AMBRIZZI, T. The role of the South Indian and Pacific oceans in South American monsoon variability. Theoretical and Applied Climatology, v. 94, n. 3/Abr, p. 125-137, 2008.

DUFEK, A. S., AMBRIZZI, T. Precipitation variability in São Paulo State, Brazil. Theoretical and Applied Climatology, 93(3-4), 167-178, 2008.

EVANGELISTA, H. et al. Evidences linking ENSO and coral growth in the SouthwesternSouth Atlantic. Climate Dynamics, v. 29, n. 7-8, p. 869-880, 1 dez. 2007.

HAYLOCK, M. R., PETERSON, T. C., ALVES, L. M., AMBRIZZI, T., ANUNCIACAO, Y. M. T., BAEZ, J., BARROS, V. R., BERLATO, M. A., BIDEGAIN, M., CORONEL, G., CORRADI, V., GARCIA, V.J., GRIMM, A. M., KAROLY, D., MARENGO, J. A., MARINO, M. B., MONCUNILL, D. F., NECHET, D., QUINTANA, J., REBELO, E., RUSTICUCCI, M., SANTOS, J. L., TREBEJO, I., VINCENT, L. A. Trends in total and extreme South American rainfall in 1960-2000 and links with sea surface temperature. Journal of Climate, v.19(8), 1490-1512, 2006.

IBGE, Instituto Brasileiro de Geografia e Estatística. Mapa do Estado de São Paulo. IBGE, 2009. Disponível em: mapas.ibge.gov.br.

KARL, T. R.; NICHOLLS, N.; GHAZI, A. Clivar/GCOS/WMO Workshop on Indices and Indicators for Climate Extremes Workshop Summary. Climatic Change, v. 42, n. 1, p. 3-7, 1 maio 1999.

KAYANO, M. T.; CAPISTRANO, V. B. How the Atlantic multidecadal oscillation (AMO) modifies the ENSO influence on the South American rainfall: AMO MODIFIES ENSO INFLUENCE. International Journal of Climatology, v. 34, n. 1, p. 162-178, jan. 2014.

MARTINELli, M. Clima do Estado de São Paulo. Confins [Online], v.8, 2010.

MEKIS, É.; VINCENT, L. A. An Overview of the Second Generation Adjusted Daily Precipitation Dataset for Trend Analysis in Canada. Atmosphere-Ocean, v. 49, n. 2, p. 163-177, 1 jun. 2011.

MONTEIRO, C. A. A Dinâmica climática e as chuvas do estado de São Paulo: estudo geográfico em forma de atlas. IG/USP. São Paulo, 1973.

MONTEIRO, C. A. O Clima e a organização do espaço no estado de São Paulo problemas e perspectivas. IGEOG/USP, n 28. São Paulo, 1976.

PSCHEIDT, I.; GRIMM, A. M. Frequency of extreme rainfall events in Southern Brazil modulated by interannual and interdecadal variability. International Journal of Climatology, v. 29, n. 13, p. 1988-2011, 2009.

QUADRO, M. D.; SILVA DIAS, M. A. F.; HERDIES, D. L.; GONCALVES, L. D. Análise climatológica da precipitação e do transporte de umidade na região da ZCAS através da nova geração de reanálises. Revista Brasileira de Meteorologia, v. 27, n. 2, p. 152-162, 2012. 
RAO, V. B. et al. An update on the rainfall characteristics of Brazil: seasonal variations and trends in 1979-2011. International Journal of Climatology, v. 36, n. 1, p. 291-302, 1 jan. 2016.

RAO, V. B.; HADA, K. Characteristics of rainfall over Brazil: Annual variations and connections with the Southern Oscillation. Theoretical and Applied Climatology, v. 42, n. 2, p. 81-91, 1 jun. 1990.

REBOITA, M. S. et al. Regimes de precipitação na América do Sul: uma revisão bibliográfica. Revista Brasileira de Meteorologia, v. 25, n. 2, p. 185-204, jun. 2010.

REBOITA, M. S. et al. Entendendo o tempo e o clima na América do Sul. Terrae Didatica, v. 8, n. 1, p. 34-50, 2012.

SANCHES, R. G. As chuvas na região de São Carlos/SP: estudo do comportamento pluviométrico a partir de dados de estações climatológicas, 1993-2014. Dissertação (mestrado). Universidade de São Paulo, 24 set. 2015.

SANCHES, R. G. et al. As chuvas na região de São Carlos/SP: análise dos valores máximos diários na série histórica, 1993-2014. Os Desafios da Geografia Física na Fronteira do Conhecimento, v. 1, n. 2017, p. 2280-2292, 25 out. 2017.

SANCHES, R. G. et al. Intense Rainfall in São Carlos/SP: Determination of Threshold Values Using Climate Indices and Their Spatio-Temporal Repercussion. American Journal of Climate Change, v. 07, p. 388, 17 ago. 2018.

SANT'ANNA NETO, J. L.; ZAVATTINI, J. A. (Org.). Variabilidade e mudanças climáticas. Eduem. Maringá, 2000.

SANTOS, C. A., SATYAMURTY, P., GOMES, O. M., DA SILVA, L. E. M. G. Variability Of Extreme Climate Indices At Rio Claro, São Paulo, Brazil. Revista Brasileira de Meteorologia. v.27, n.4, 395 - 400, 2012.

SANTOS, C. A. C. Recent Changes In Temperature And Precipitation Extremes In An Ecological Reserve In Federal District, Brazil. Revista Brasileira de Meteorologia, v. 29, n.1, 13-20, 2014.

SANTOS, C. A. C. DOS; RAMOS, A. R. D. Avaliação dos eventos extremos de precipitação no estado do Piauí. Agrometeoros, v. 25, n. 1, 21 mar. 2018.

SANTOS, B. C. dos; SOUZA, P. H. de; VECCHIA, F. A. da S. A caracterização da precipitação do ano hidrológico de 2013-2014 na região de São Carlos/SP e sua repercussão no espaço geográfico. Revista Brasileira de Climatologia, v. 21, n. 0, 15 set. 2017.

SANTOS, E. M. Estudo da variabilidade e mudanças climáticas na região de Manaus. 2010. Dissertação (Mestrado) - Instituto Nacional de Pesquisas da Amazônia, Universidade do Estado do Amazonas. Amazonas, 2010. 
SILVEIRA, L. M. DA. Os sistemas atmosféricos e a variação do tempo em Maringá, Estado do Paraná, Brasil - DOI: 10.4025/actascitechnol.v28i1.1290. Acta Scientiarum. Technology, v. 28, n. 1, p. 79-84, 20 mar. 2008.

STEPHENSON, T. S. et al. Changes in extreme temperature and precipitation in the Caribbean region, 1961-2010. International Journal of Climatology, v. 34, n. 9, p. 29572971, 1 jul. 2014.

TEIXEIRA, M. DA S.; SATYAMURTY, P. Trends in the Frequency of Intense Precipitation Events in Southern and Southeastern Brazil during 1960-2004. Journal of Climate, v. 24, n. 7, p. 1913-1921, 27 jan. 2011.

VÁSQUEZ P., I. L. et al. Historical analysis of interannual rainfall variability and trends in southeastern Brazil based on observational and remotely sensed data. Climate Dynamics, v. 50, n. 3, p. 801-824, 1 fev. 2018.

ZANDONADI, L. As chuvas na bacia hidrográfica do rio Paraná, Brasil: um estudo do ritmo climático e algumas considerações sobre a vazão hídrica. 2013. 205 p. Tese (doutorado) - Universidade Estadual Paulista, Instituto de Geociências e Ciências Exatas, 2013. Disponível em: <http://hdl.handle.net/11449/104299>.

ZANDONADI, L.; ACQUOTTA, F.; FRATIANNI, S.; ZAVATTINI, J. A. Changes in precipitation extremes in Brazil (Paraná River Basin). Theoretical and Applied Climatology, v. 123, n. 3, p. 741-756, 2016.

ZAVATTINI, J. A.; BOIN, M. N. Climatologia geográfica: teoria e prática de pesquisa. Editora Alínea. Campinas, 2013.

ZHANG, X.; YANG, F.; RClimDex (1.0) User Guide. Climate Research Branch Environment Canada: Downsview, Ontario, Canada, 2004.

ZILLI, M. T. et al. A comprehensive analysis of trends in extreme precipitation over southeastern coast of Brazil. International Journal of Climatology, v. 37, n. 5, p. 22692279, 1 abr. 2017.

ZWIERS, F. W., VON STORCH, H. (2004). On the role of statistics in climate research. International Journal of Climatology, 24(6), 665-680, 2004. 


\section{Rafael Grecco Sanches}

Mestre em Ciências da Engenharia Ambiental pela Escola de Engenharia de São Carlos (EESC) da Universidade de São Paulo (USP). Graduado em Geografia pela Universidade Sagrado Coração, Bauru/SP. Atualmente é doutorando em Ciências da Engenharia Ambiental da EESC/USP na área de Climatologia Aplicada.

Universidade de São Paulo - Av. Trabalhador São-carlense, 400 - Pq. Arnold Schimidt CEP 13566-590 - São Carlos/SP - Brasil

E-mail: rafagsanches@usp.br

\section{Francisco Arthur da Silva Vecchia}

Doutor em Ciências, Programa de Pós-Graduação em Geografia, Faculdade de Filosofia, Letras e Ciências Humanas. Graduação em Engenharia de Produção, Escola de Engenharia de São Carlos. Atualmente é Professor Associado A3 (Livre Docente) da Escola de Engenharia de São Carlos da Universidade de São Paulo.

Universidade de São Paulo - Av. Trabalhador São-carlense, 400 - Pq. Arnold Schimidt CEP 13566-590 - São Carlos/SP - Brasil

E-mail: fvecchia@usp.br

\section{Paulo Henrique de Souza}

Doutor em Ciências da Engenharia Ambiental pela Escola de Engenharia de São Carlos da Universidade de São Paulo. Graduação em Geografia pela Universidade Estadual Paulista Júlio de Mesquita Filho. Atualmente exerce pesquisa e ensino na Universidade Federal de Alfenas UNIFAL-MG.

Universidade Federal de Alfenas UNIFAL-MG - Avenida Jovino Fernandes Sales, 2600

- Bairro Santa Clara - Alfenas/MG - CEP 37.133-840.

Recebido para publicação em dezembro de 2018 Aprovado para publicação em novembro de 2019 